\title{
EFFECT OF PRIMARY PREVENTIVE EXAMINATION WITH CARDIORESPIRATORY FITNESS TEST
}

\author{
Šárka Kunzová', 2 , Jindřich Fiala ${ }^{1,2}$, Pavel Řimák ${ }^{1,2}$, Petr Kaňovský \\ ${ }^{1}$ Department of Public Health, Faculty of Medicine, Masaryk University, Brno, Czech Republic \\ ${ }^{2}$ International Clinical Research Centre, St. Anne's University Hospital, Brno, Czech Republic
}

\section{SUMMARY}

Objective: The aim of this study was to explore the potential of free comprehensive primary preventive examination (CPPE) combined with cardiorespiratory fitness (CRF) test in terms of its attractiveness for general population and moreover to evaluate the induced behavioural changes. The main focus was on physical activity behaviour (PA).

Methods: In 2009-2013, 250 people (100 men, 150 women) aged 18-65 years were examined. CPPE included assessment of health status and lifestyle, CRF test and individualized counselling. Expectations, reasons and motivations for participating were recorded. The sample was evaluated in terms of age, gender, lifestyle, body mass index, body fat percentage, CRF, and health characteristics. Evaluation according to subjective benefits, perceived effects on health and lifestyle was performed after six months using electronic feedback questionnaires ( $F Q$ ). Comparison was made within groups formed according to the reported increase in PA.

Results: People aged $18-39$ years accounted for $72.8 \%$ of the sample; mean age $34.4 \pm 11.0$ years; $40.0 \%$ were men. Behavioural and health risks were lower in comparison with the general Czech population, but at least 1 of 5 assessed risk factors was present in $88.8 \%$ (low fruit and vegetable consumption $74.8 \%$, low physical activity level (PAL) $45.6 \%$, smoking 19.6\%, risky alcohol use $18.8 \%$, and stress load $10.4 \%$ ). The most represented category of CRF was "endurance-trained" (both genders). CPPE was perceived as a source of information concerning health, CRF and lifestyle. $40.0 \%$ of men and $30.7 \%$ of women were focused on improvement in CRF. The response rate of $F Q$ was $75.6 \%$. Individuals with low PAL and low CRF provided feedback less often $(p<0.05)$. In terms of perceived effect, $84.1 \%$ of the respondents implemented some kind of behavioural change; $60.9 \%$ reported increase in PA, but only 38.1\% reported maintaining improvement in PA after 6 months. A higher proportion of reported lasting changes in PA occurred in subjects who were overweight/obese and in those with low CRF. Participants with low PAL and higher number of lifestyle risks more likely increased their PA only temporarily. Improvement in PA was associated with reported changes in diet $(p<0.001)$. In the group of respondents there was an increase in self-perceived PA (SPA) compared to the baseline $(p=0.001)$. Moreover, individuals who reported increase in PA showed improvement in subjectively perceived health.

Conclusion: The testing of CRF appears to be a promising motivating factor for going through the intervention, especially for younger people and men. CPPE is effective at the individual level in terms of providing information and initiating behavioural changes in PA. However, this type of intervention is less attractive and less effective for individuals with a higher behavioural risk profile.

Key words: primary prevention, lifestyle, cardiorespiratory fitness test, physical activity, young adults, behavioural change

Address for correspondence: Š. Kunzová, Department of Public Health, Faculty of Medicine, Masaryk University, Kamenice 753/5, 62500 Brno, Czech Republic. E-mail: kunzova.s@seznam.cz

https://doi.org/10.21101/cejph.a4415

\section{INTRODUCTION}

A healthy lifestyle is the basis for the prevention of noncommunicable diseases such as cardiovascular diseases (CVDs), obesity, diabetes, and certain types of cancer. Modification of adverse behavioural characteristics is highly important, not only in at risk population groups, but also as a primary prevention in young and healthy individuals (1). Public health and individual efforts are needed to improve the adoption and maintenance of a healthy lifestyle in these hard to reach population groups for health-related interventions. There is the evidence that maintaining a healthy lifestyle throughout young adulthood is strongly associated with a favourable health outcome in middle age, e.g. low CVDs risk profile (2). Different lifestyle factors are longacting and interacting. Therefore, lifestyle assessments should be made comprehensively and in the context of health status. Health enhancing PA has beneficial effects on both physical and mental health and is associated with long term health advantages (1). High levels of recreational PA may improve sleep quality (3). Both, healthy and unhealthy behaviours tend to cluster and therefore the change in one area of lifestyle can influence the others (4). Thus, it may be that improvement in PA creates synergistic improvements in other behaviours. Furthermore, PA can serve as a compensation for unhealthy choices, especially in young adults (5).

While the importance of a healthy lifestyle in public health is well established, PA in the Czech Republic has continued to decline $(6,7)$. Incorrect perception of body weight and overestimation of PA forms one of the potential barriers in PA promotion. Overestimation is associated with male gender $(8,9)$ and favourable indicators of relative slimness and general health $(9$, 
10). Systematic review of trials of PA and dietary counselling to prevent CVDs in adults with minimum follow-up of six months confirmed that the intervention intensity, risk profile in the population and use of volunteer participants predicted bigger effect. Medium (31-360 minutes) to high intensity (>360 minutes) counselling resulted in favourable changes in health outcomes and behavioural characteristics (11). Combined behavioural interventions aimed at simultaneous modification (PA and dietary habits) are more effective in the long term in comparison to single component programmes (12).

Many high-intensity interventions in research required resources that may not be available or paid for in the current healthcare system (11). Therefore, interventions that would be easily accessible and applicable in routine practice are needed. It is also worth questioning the attractiveness of these measures for population groups with a low interest in prevention, such as men and young people who still do not feel the health consequences of their risk behaviours. Young adults who attempt to achieve a healthy lifestyle prefer certain promotion strategies, in particular, those that are more affordable, relate to personalized advice or support and easily accessed advice from a range of media sources (5). In general, men are more often engaged in risk behaviours, are less interested in clinical or preventive health care (13) and tend to express unrealistic self-assessment $(8,9)$. On the other hand, men exhibit more favourable characteristics in terms of PA compared to women $(6,14,15)$, more engage in leisure-time physical activity (16), and we might think they have a closer relationship to sport in general. Therefore, an offer of preventive examination with CRF test may be a natural way to convey other areas of health prevention to those interested in this type of testing. Moreover, such testing helps participants increase awareness about their PA and current health status.

Within the low-threshold primary preventive programme for the public, conducted at the Department of Public Health, Faculty of Medicine, Masaryk University Brno, the free comprehensive primary preventive examination (CPPE) was offered to the general public through Internet websites on a server of the Masaryk University. The programme links to the previously realized projects $(17,18)$. The three specific objectives of this study were to explore the potential of such intervention in terms of its attractiveness for the general population with respect to age, gender and behavioural characteristics; to consider the inclusion of the CRF test as a motivational factor for participating in the examination and lifestyle adjustment; and to evaluate the subjective effect of this medium-intensity intervention in the time frame of six months.

\section{MATERIALS AND METHODS}

\section{Study Design and Subjects}

The target group was the general adult population. Volunteers came for CPPE on the basis of public offer on the Internet or references from individuals already examined. In 2009-2013, a total number of 250 volunteers ( $40 \%$ of men) aged $18-65$ years were examined by healthcare professionals.

The mean age of the sample was $34.4 \pm 11.0$ years. People aged 18-39 accounted for $72.8 \%$ of the sample.

The university degree had $67.6 \%$ of participants. There were $24.8 \%$ of students, $67.2 \%$ of economically active people, $5.2 \%$ of the sample were women on a maternity leave, $2.0 \%$ retired people, and $0.8 \%$ people with disability.

The intervention included evaluation of lifestyle risk factors, measurement of blood pressure and anthropometric characteristics, assessment of body composition, and CRF. Based on the results individualized counselling was made on the principles of motivational interviewing according to participants' readiness to change in order to influence behavioural risks $(1,19)$. The total duration of CPPE ranged from 90 to 120 minutes. All participants provided a written consent to participate. A feedback questionnaire was sent via e-mail to every participant six months after his/her examination.

\section{Baseline Examination}

Baseline questionnaire included socio-demographic characteristics, dietary habits, PA, alcohol consumption, tobacco smoking, stress load, subjectively perceived health, reasons, motivation, and expectations for participating in CPPE.

A dietary assessment of basic food groups was performed using the semi-quantitative food frequency questionnaire (Czech version modified by J. Fiala, 1998) (20). For the assessment, we used data on daily intake of fruit and vegetables (F\&V). According to the current Dietary guidelines for the Czech Republic (21), daily intake lower than 6 servings of F\&V was defined as a behavioural risk (low F\&V).

Alcohol use with a daily consumption of $>10 \mathrm{~g}$ for women and $>20 \mathrm{~g}$ for men was considered high and defined as a behavioural risk $(1,21)$. Smoking was evaluated by medical history using both active and passive exposure to tobacco smoke. Daily or occasional smoking and very frequent passive exposure was defined as a behavioural risk.

Physical activity level (PAL) in terms of recreational physical activity during last 4-8 weeks was assessed in terms of frequency and intensity by medical history. Three groups of PAL were defined: low ( $<3$ times per week of at least moderate-intensity physical activity) defined as behavioural risk; medium (at least 3 times per week of at least moderate-intensity exercise, but criterion for high PAL is not reached); high (to perform moderateintensity exercise more than 30 minutes daily in 5 days per week) or vigorous-intensity exercise (more than 20 minutes daily in 3 days per week) $(1,22)$.

Self-perceived physical activity level (SPA) was based on direct self-assessment and classified on the seven-item scale ranged 1-7 (very poor, poor, low, moderate, more, high, very high).

Stress load (SL) was assessed as the sum of three subjective items (frequency of exposure, perceived level of stress, perceived resistance) with total score ranged 0-20. Three categories of SL were defined: low (score 0-9); increased (10-15); excessive $(\geq 16)$ defined as behavioural risk.

Lifestyle cumulative risk score (LCR) was calculated as a sum of present risk factors (low F\&V consumption, low PAL, high alcohol use, smoking, excessive SL) ranged 0-5. Subjectively perceived health (SPH) was assessed on a five-item scale ranged 1-5 (very poor, poor, satisfactory, good, very good).

\section{Measurements}

Casual blood pressure (BP) was examined on a calibrated digital tonometer Omron M6 in a sitting position. A mean systolic and 
diastolic BP was calculated (from the 2nd and 3rd standardized measurement after 8 and 11 minutes of rest). Weight and height were measured according to standards with subjects wearing light clothing. Body mass index (BMI) was calculated $(1,23)$. The device Bodystat 1500 (bioelectrical impedance body composition analyser single frequency) was used to perform body composition analysis and evaluate body fat percentage (BF\%) with respect to age. The applied method is unified and the measurement and evaluation was performed according to standards set in the manual of the device (24).

For an accurate identification of anthropometric risk we take into account both, results of body composition analysis and the BMI classification $(1,21)$. Total anthropometric risk score (TAR) categories were defined: TAR 0 (BMI $<30 \mathrm{~kg} / \mathrm{m}^{2}$ and normal $\mathrm{BF} \%$ ); TAR 1 (BMI $<25 \mathrm{~kg} / \mathrm{m}^{2}$ and increased BF\%); TAR 2 (BMI 25.0-29.99 kg/m² and increased BF\%); and TAR 3 (BMI $\geq 30 \mathrm{~kg} / \mathrm{m}^{2}$ ).

Cardiorespiratory fitness (CRF) assessment was performed using the indirect method determining $\mathrm{VO}^{2}$ max on a bicycle ergometer Ergoline (model 200K, software Ergoselect). Results were categorized according to Seliger and Bartunek (25): CRF 1 (low); CRF 2 (below average); CRF 3 (average); CRF 4 (above the average) - endurance trained.

\section{Feedback Questionnaire}

Six months after the baseline an electronic feedback questionnaire (FQ) was sent to every participant. If there was no response, the request to answer FQ was sent 2 more times at intervals of 2-4 weeks. FQ included questions to evaluate baseline examinations, current SPH and SPA, subjective benefits/effects and realized lifestyle changes. The implemented behavioural change was assessed according to subjectively reported changes - improvements in terms of PA, nutrition and other lifestyle areas (alcohol, smoking, sleep) and evaluated according to their duration as "temporary" (1-5 months) and "lasting" (6 months or longer).

\section{Data Analysis}

For descriptive statistics and data analysis the statistical package IBM SPSS Statistics 22 was used (26).

For discrete variables absolute and relative frequencies were calculated, for continuous variables averages and standard deviations were calculated. Differences were considered statistically significant at $p<0.050$. Testing differences in frequencies between groups and relationships of monitored parameters was performed using Chi-square $\left(\chi^{2}\right)$ test and contingency tables. Three age categories were defined: young (18-30 years), middle age (31-50 years), and older age ( $\geq 51$ years). Comparison was performed within groups formed according to the reported increase in PA (lasting, temporary, no change). To examine whether CPPE and subsequent changes in PA behaviour led to a change in self-assessment parameters (SPA, SPH), the deterioration, stagnation or improvement of these parameters were considered (baseline vs. FQ), and groups of respondents according to the duration of reported increase in PA were compared using $\chi^{2}$ test. Secondly, paired t-test was used for comparison of self-assessment parameters (SPA, SPH) baseline vs. FQ in the whole group of respondents.

\section{RESULTS}

Socio-demographic, behavioural, anthropometric and health characteristics of the sample are shown in Table 1. A personal history of CVDs or metabolic diseases had $8.4 \%$ of the subjects $(n=21)$ (hypertension: $n=16(6.4 \%)$; dyslipidaemia: $n=6(2.4 \%)$; coronary heart disease: $n=2(0.8 \%)$; diabetes mellitus: $n=1$ $(0.4 \%))$. Women were more likely to have low and normal weight (mean BMI $=24.3 \pm 4.9 \mathrm{~kg} / \mathrm{m}^{2}$; mean $\mathrm{BF} \%=28.9 \pm 7.4$ ). Men were more likely to be overweight and obese (mean BMI $=26.1 \pm 4.3$ $\mathrm{kg} / \mathrm{m}^{2}$; mean $\left.\mathrm{BF} \%=18.7 \pm 6.7\right)$. Overall, $22.0 \%$ of men and $0.7 \%$ of women were athletes with normal $\mathrm{BF} \%$, but overweight according to BMI due to increased muscle mass. In terms of TAR, $\mathrm{TAR}=0$ had more than half of the subjects, more often men. Overall, $14.0 \%$ of the sample (men $3.0 \%$, women $21.3 \%$ ) with normal weight according to BMI had increased $\mathrm{BF} \%$. At least one of five assessed lifestyle risk factors was present in $88.8 \%$ of the examined. The most common was low F\&V consumption, low PAL was found in $45.6 \%$. CRF was measured in $94.4 \%$ of the sample $(\mathrm{n}=236)$ (men: mean $\mathrm{VO}^{2} \max =41.7 \pm 8.0 \mathrm{ml} / \mathrm{kg}$ / min; women: mean $\mathrm{VO}^{2} \max =32.0 \pm 7.1 \mathrm{ml} / \mathrm{kg} / \mathrm{min}$ ). Lower than average CRF (category 1 +2 ) had a quarter of men and more than a third of women. The most represented category was "endurancetrained" (both genders).

Relationships of PAL, TAR, age, and CRF are presented in Tables 2 and 3. Low PAL was found most frequently in middle age group among men and in young and middle age groups among women; the older age group was rather of medium or high PAL. PAL showed a positive correlation with CRF and negative correlation with TAR; the higher was TAR, the higher was proportion of poor CRF and low PAL. Participants with TAR 1 had the highest proportion of low PAL, but in terms of CRF they were frequently above the average. TAR grew with age in both genders.

Reasons for passing CPPE and expectations are presented in Table 4. The results show that CPPE was primarily seen as an information resource about current health status and CRF. There were found interesting but mostly non-significant gender differences. Women were more often dissatisfied with their health $(p=0.107)$ and weight $(p=0.071)$ and wanted to motivate another person (partner, friend, relative) towards lifestyle change $(p=0.006)$. Men often came with concerns about their health $(p=0.086)$ and wanted to improve their CRF $(p=0.128)$. Women more frequently expected advice and information about nutrition $(p=0.044)$ and weight loss $(p=0.023)$, as well as support for a decision to change $(\mathrm{p}=0.095)$.

Feedback questionnaire was completed by $75.6 \%$ of the people examined. In the group of respondents $(n=189$; men $42.9 \%$, women $57.1 \%$ ), mean age was $34.0 \pm 11.4$ years; the university degree had $71.4 \%$. Total of 61 persons (men $31.1 \%$, women $68.9 \%$; mean age $35.6 \pm 9.55$ years) did not respond to the email or provide a feedback. There was non-significantly higher proportion of men in the group of respondents $(\mathrm{p}=0.105)$. In a comparison with respondents (not presented in Tables), non-respondents had lower SPA $(p=0.004)$ and a higher presence of both low PAL $(\mathrm{p}=0.016)$ and low CRF $(\mathrm{p}=0.012)$.

There were no significant gender differences in perceived benefits or realized lifestyle changes in the group of respondents (Table 5). CPPE was mostly rated as a source of new information and verification of the known facts, Moreover, respondents were 
Table 1. Sample characteristics and lifestyle risk factors by gender

\begin{tabular}{|c|c|c|c|c|c|}
\hline \multicolumn{2}{|l|}{ Variable } & $\begin{array}{c}\text { Total } \\
(\mathrm{n}=250) \\
\%\end{array}$ & $\begin{array}{c}\text { Men } \\
(n=100) \\
\%\end{array}$ & $\begin{array}{c}\text { Women } \\
(\mathrm{n}=150) \\
\%\end{array}$ & $p$ value* \\
\hline \multirow{3}{*}{ Age (years) } & $18-30$ & 45.2 & 42.0 & 47.3 & \multirow{3}{*}{0.266} \\
\hline & $31-50$ & 43.2 & 49.0 & 39.3 & \\
\hline & $51-65$ & 11.6 & 9.0 & 13.3 & \\
\hline \multirow{3}{*}{ Education } & Secondary vocational & 2.4 & 4.0 & 1.3 & \multirow{3}{*}{0.005} \\
\hline & Secondary school & 30.0 & 19.0 & 37.3 & \\
\hline & University degree & 67.6 & 77.0 & 61.3 & \\
\hline \multirow{5}{*}{ Presence of behavioural risk factors } & Low F\&V consumption & 74.8 & 83.0 & 69.3 & 0.015 \\
\hline & Low physical activity level & 45.6 & 36.0 & 52.0 & 0.013 \\
\hline & High alcohol use & 18.8 & 26.0 & 14.0 & 0.017 \\
\hline & Smoking & 19.6 & 21.0 & 18.7 & 0.649 \\
\hline & Excessive stress load & 10.4 & 12.0 & 9.3 & 0.499 \\
\hline \multirow{5}{*}{ Lifestyle cumulative risk score } & 0 & 11.2 & 9.0 & 12.7 & \multirow{5}{*}{0.134} \\
\hline & 1 & 32.0 & 36.0 & 29.3 & \\
\hline & 2 & 38.4 & 31.0 & 43.3 & \\
\hline & 3 & 13.6 & 17.0 & 11.3 & \\
\hline & 4 and 5 & 4.8 & 7.0 & 3.3 & \\
\hline \multirow{3}{*}{ Physical activity level (PAL) } & Low & 45.6 & 36.0 & 52.0 & \multirow{3}{*}{0.045} \\
\hline & Medium & 28.4 & 33.0 & 25.3 & \\
\hline & High & 26.0 & 31.0 & 22.7 & \\
\hline \multirow{6}{*}{ Self-perceived physical activity } & 1 (very poor) & 8.8 & 7.0 & 10.0 & \multirow{6}{*}{0.002} \\
\hline & 2 (poor) & 16.0 & 17.0 & 15.3 & \\
\hline & 3 (low) & 20.8 & 17.0 & 23.3 & \\
\hline & 4 (moderate) & 26.4 & 18.0 & 32.0 & \\
\hline & 5 (more) & 21.2 & 28.0 & 16.7 & \\
\hline & 6 (high) and 7 (very high) & 6.8 & 13.0 & 2.7 & \\
\hline \multirow{5}{*}{ Subjectively perceived health } & 1 (very poor) & 0.4 & 0.0 & 0.7 & \multirow{5}{*}{0.638} \\
\hline & 2 (poor) & 9.6 & 9.0 & 10.0 & \\
\hline & 3 (satisfactory) & 28.0 & 24.0 & 30.7 & \\
\hline & 4 (good) & 45.2 & 50.0 & 42.0 & \\
\hline & 5 (very good) & 16.8 & 17.0 & 16.7 & \\
\hline \multirow{4}{*}{ BMI $\left(\mathrm{kg} / \mathrm{m}^{2}\right)$} & $<18,5$ & 3.2 & 1.0 & 4.7 & \multirow{4}{*}{0.002} \\
\hline & $18.5-24.99$ & 53.6 & 42.0 & 61.3 & \\
\hline & $25.0-29.99$ & 28.8 & 40.0 & 21.3 & \\
\hline & $\geq 30$ & 14.4 & 17.0 & 12.7 & \\
\hline Body fat (\%) & High body fat \% & 48.0 & 38.0 & 54.7 & 0.010 \\
\hline \multirow{4}{*}{ Total anthropometric risk score $(\mathrm{TAR})^{\star *}$} & 0 & 52.0 & 62.0 & 45.3 & \multirow{4}{*}{$<0.001$} \\
\hline & 1 & 14.0 & 3.0 & 21.3 & \\
\hline & 2 & 19.6 & 18.0 & 20.7 & \\
\hline & 3 & 14.4 & 17.0 & 12.7 & \\
\hline Blood pressure (mmHg) & $\geq 140 / 90$ & 14.8 & 21.0 & 10.7 & 0.024 \\
\hline \multirow{4}{*}{ Cardiorespiratory fitness (CRF) ${ }^{* * *}$} & 1 (low) & 16.9 & 14.7 & 18.4 & \multirow{4}{*}{0.192} \\
\hline & 2 (below the average) & 15.7 & 10.5 & 19.1 & \\
\hline & 3 (average) & 20.3 & 24.2 & 17.7 & \\
\hline & 4 (above the average) & 47.0 & 50.5 & 44.7 & \\
\hline
\end{tabular}


Table 2. Physical activity level in relation to age, cardiorespiratory fitness and total anthropometric risk score

\begin{tabular}{|c|c|c|c|c|c|}
\hline & & & activity le & & \\
\hline Variable & & Low & Medium & High & $p$ value* \\
\hline Age (years) & $18-30$ & 40.4 & 43.7 & 55.4 & \\
\hline & $31-50$ & 53.5 & 38.0 & 30.8 & 0.008 \\
\hline & $51-65$ & 6.1 & 18.3 & 13.8 & \\
\hline Cardiorespiratory fitness (CRF) $)^{\star \star *}$ & 1 (low) & 27.4 & 6.0 & 11.1 & \\
\hline & 2 (below the average) & 24.5 & 10.4 & 6.3 & 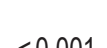 \\
\hline & 3 (average) & 24.5 & 20.9 & 12.7 & 0.001 \\
\hline & 4 (above the average) & 23.6 & 62.7 & 69.8 & \\
\hline Total anthropometric risk score $(\mathrm{TAR})^{\star *}$ & 0 & 43.9 & 50.7 & 67.7 & \\
\hline & 1 & 20.2 & 12.7 & 4.6 & 041 \\
\hline & 2 & 20.2 & 22.5 & 15.4 & 0.041 \\
\hline & 3 & 15.8 & 14.1 & 12.3 & \\
\hline
\end{tabular}

${ }^{*} \mathrm{p}$ value refers to $\mathrm{X}^{2}$ test over all answering categories; **TAR: 0 (BMI < 30 and normal BF\%), 1 (BMI < 25 and increased $\left.\mathrm{BF} \%\right), 2$ (BMI $25.0-29.99$ and increased $\mathrm{BF} \%$ ), $3(\mathrm{BMI} \geq 30)$; ${ }^{* *}$ percentages related to the number of subjects who attended CRF test in each PAL category

Table 3. Total anthropometric risk score in relation to age, cardiorespiratory fitness and physical activity level

\begin{tabular}{|c|c|c|c|c|c|c|}
\hline \multirow{2}{*}{\multicolumn{2}{|c|}{ Variable }} & \multicolumn{4}{|c|}{ Total anthropometric risk score $(\mathrm{TAR})^{* *}$} & \multirow[b]{2}{*}{$p$ value } \\
\hline & & \multirow{2}{*}{$\begin{array}{c}0 \\
(n=130) \\
\% \\
53.8\end{array}$} & \multirow{2}{*}{$\begin{array}{c}1 \\
(\mathrm{n}=35) \\
\%\end{array}$} & \multirow{2}{*}{$\left.\begin{array}{c}2 \\
(n=49) \\
\%\end{array}\right)$} & \multirow{2}{*}{$\begin{array}{c}\begin{array}{c}3 \\
(n=36) \\
\%\end{array} \\
22.2\end{array}$} & \\
\hline Age (years) & $18-30$ & & & & & \multirow{3}{*}{0.004} \\
\hline & $31-50$ & 40.8 & 37.1 & 44.9 & 55.6 & \\
\hline & $51-65$ & 5.4 & 14.3 & 18.4 & 22.2 & \\
\hline \multirow{4}{*}{$\begin{array}{l}\text { Cardiorespiratory } \\
\text { fitness }(\mathrm{CRF})^{\star \star \star}\end{array}$} & 1 (low) & 2.5 & 15.2 & 29.2 & 52.9 & \multirow{4}{*}{$<0.001$} \\
\hline & 2 (below the average) & 14.9 & 21.2 & 12.5 & 17.6 & \\
\hline & 3 (average) & 17.4 & 24.2 & 27.1 & 17.6 & \\
\hline & 4 (above the average) & 65.3 & 39.4 & 31.3 & 11.8 & \\
\hline \multirow{3}{*}{$\begin{array}{l}\text { Physical activity } \\
\text { level (PAL) }\end{array}$} & Low & 38.5 & 65.7 & 46.9 & 50.0 & \multirow{3}{*}{0.041} \\
\hline & Medium & 27.7 & 25.7 & 32.7 & 27.8 & \\
\hline & High & 33.8 & 8.8 & 20.4 & 22.2 & \\
\hline
\end{tabular}

${ }^{*} \mathrm{p}$ value refers to $\mathrm{X}^{2}$ test over all answering categories; **TAR: 0 (BMl < 30 and normal BF\%), 1 (BMI < 25 and increased $\left.\mathrm{BF} \%\right), 2$ (BMI $25.0-29.99$ and increased $\mathrm{BF} \%$ ), $3(\mathrm{BMI} \geq 30)$; ${ }^{* * *}$ percentages are related to the number of subjects who attended CRF test in each TAR category

directly motivated (36.5\%) or supported $(24.9 \%)$ in previous decision to make a change. Increasing PA in total reported $60.9 \%$ but only $38.1 \%$ of respondents reported six months lasting increase in PA. Dietary changes reported $65.6 \%$ of respondents but lasting improvement only $31.2 \%$ of respondents.

A comparison according to the duration of reported changes in PA among respondents is presented in Tables 6 and 7. Duration of implemented changes was probably linked to the presence of low PAL $(\mathrm{p}<0.001)$. People with low PAL often made changes, but they were with a higher proportion of temporary ones. If we assume that non-respondents did not implement any change, approximately both, half of tested with and without low PAL increased their PA, but the sustainability of this change was higher in the group without this behavioural risk. Participants with low CRF had a higher proportion of lasting changes. With an increase in TAR the permanence of changes increased and no-change was decreasing. Overweight and obese participants therefore responded by increasing PA. Lasting changes were more frequent than temporary ones in all categories of TAR except TAR 1 , where the highest proportion of temporary changes were observed. In terms of LCR it was evident that people without lifestyle risks (LCR 0) were more often able to realize lasting improvement in PA, whereas people with a high LCR frequently increased their PA only temporarily. In terms of SPA, individuals, who were average or above average in this subjective parameter in baseline, had the highest proportion of no change. But if they increased their PA, this change often had a more lasting effect. Those, who were below average in SPA, had more often implemented changes, but with a higher proportion of temporary ones. Reported improvements in PA were associated with the implementation of changes in nutrition $(p<0.001)$. 
Table 4. Reasons/motivations for passing examination and expectations by gender

\begin{tabular}{|c|c|c|c|c|c|}
\hline Variable & & $\begin{array}{c}\text { Total } \\
\mathrm{N}=(250)\end{array}$ & $\begin{array}{c}\text { Men } \\
(n=100)\end{array}$ & $\begin{array}{l}\text { Women } \\
(n=150)\end{array}$ & $p$ value* \\
\hline & To evaluate the health status/fitness & 78.0 & 75.0 & 80.0 & 0.350 \\
\hline & Dissatisfaction/effort to feel better & 55.2 & 49.0 & 59.3 & 0.107 \\
\hline & To obtain information (health/lifestyle) & 38.4 & 41.0 & 36.7 & 0.490 \\
\hline & To improve fitness & 34.4 & 40.0 & 30.7 & 0.128 \\
\hline & Effort to reduce excess weight & 33.6 & 27.0 & 38.0 & 0.071 \\
\hline Reason and motivation & Promotion of implemented changes & 20.4 & 20.0 & 20.7 & 0.898 \\
\hline & Health concerns & 18.8 & 24.0 & 15.3 & 0.086 \\
\hline & To motivate another person to change & 9.2 & 3.0 & 13.3 & 0.006 \\
\hline & Curiosity & 4.8 & 5.0 & 4.7 & 0.904 \\
\hline & Effort to quit smoking & 1.2 & 1.0 & 1.3 & 0.813 \\
\hline & Other & 4.4 & 3.0 & 5.3 & 0.378 \\
\hline & Evaluation of cardiorespiratory fitness & 81.2 & 82.0 & 80.7 & 0.792 \\
\hline & Health assessment & 65.6 & 67.0 & 64.7 & 0.704 \\
\hline & Advice to improve health & 54.0 & 50.0 & 56.7 & 0.300 \\
\hline Expectation & New information about nutrition & 34.4 & 27.0 & 39.3 & 0.044 \\
\hline & Support of the decision to change lifestyle & 22.4 & 17.0 & 26.0 & 0.095 \\
\hline & Advice how to lose weight & 21.2 & 14.0 & 26.0 & 0.023 \\
\hline & Other & 3.2 & 4.0 & 2.7 & 0.557 \\
\hline
\end{tabular}

${ }^{*} \mathrm{p}$ value refers to $\mathrm{X}^{2}$ test of presence of reasons/motivations/expectations within groups by gender. When answering questions about reasons/motivation/expectation, multiple choices could have been selected.

Table 5. Perceived benefits and reported lifestyle changes by gender within the group of respondents

\begin{tabular}{|c|c|c|c|c|c|c|}
\hline \multicolumn{3}{|l|}{ Variable } & $\begin{array}{c}\text { Total } \\
\text { (n=189) } \\
\%\end{array}$ & $\begin{array}{c}\text { Men } \\
(n=81) \\
\%\end{array}$ & $\begin{array}{c}\text { Women } \\
(n=108) \\
\%\end{array}$ & p value* \\
\hline \multirow{8}{*}{ Perceived benefits } & \multicolumn{2}{|c|}{ I obtained new information } & 84.7 & 86.4 & 83.3 & 0.560 \\
\hline & \multicolumn{2}{|c|}{ I checked what I already know } & 45.0 & 40.7 & 48.1 & 0.311 \\
\hline & \multicolumn{2}{|c|}{ I decided to make lifestyle changes } & 36.5 & 39.5 & 34.3 & 0.458 \\
\hline & \multicolumn{2}{|c|}{ Promotion of motivation to change } & 24.9 & 23.5 & 25.9 & 0.697 \\
\hline & \multicolumn{2}{|c|}{ I became interested in prevention } & 15.9 & 18.5 & 13.9 & 0.389 \\
\hline & \multicolumn{2}{|c|}{ I checked that I live healthily } & 3.7 & 1.2 & 5.6 & 0.120 \\
\hline & \multicolumn{2}{|c|}{ I began to worry about my health } & 4.8 & 3.7 & 5.6 & 0.554 \\
\hline & \multicolumn{2}{|c|}{ Examinations had no benefit } & 0.5 & 0.0 & 0.9 & 0.385 \\
\hline \multirow{11}{*}{$\begin{array}{l}\text { Reported lifestyle } \\
\text { changes }\end{array}$} & \multirow{2}{*}{ Change in total } & No change & 15.9 & 17.3 & 14.8 & \multirow{2}{*}{0.646} \\
\hline & & Yes & 84.1 & 82.7 & 85.2 & \\
\hline & \multirow{3}{*}{$\begin{array}{l}\text { Increasing } \\
\text { physical activity } \\
\text { behaviour }\end{array}$} & No change & 39.2 & 46.9 & 33.3 & \multirow{3}{*}{0.167} \\
\hline & & Temporary & 22.8 & 19.8 & 25.0 & \\
\hline & & Lasting & 38.1 & 33.3 & 41.7 & \\
\hline & \multirow{3}{*}{$\begin{array}{l}\text { Change in } \\
\text { nutrition }\end{array}$} & No change & 34.4 & 37.0 & 32.4 & \multirow{3}{*}{0.775} \\
\hline & & Temporary & 34.4 & 32.1 & 36.1 & \\
\hline & & Lasting & 31.2 & 30.9 & 31.5 & \\
\hline & \multirow{3}{*}{$\begin{array}{l}\text { Other lifestyle } \\
\text { changes }\end{array}$} & No change & 91.0 & 86.4 & 94.4 & \multirow{3}{*}{0.083} \\
\hline & & Temporary & 4.8 & 8.6 & 1.9 & \\
\hline & & Lasting & 4.2 & 4.9 & 3.7 & \\
\hline
\end{tabular}

${ }^{*} p$ value refers to $x^{2}$ test over all answering categories within groups by gender or to $x^{2}$ test of the presence of perceived benefits within groups by gender. When answering questions about perceived benefits, multiple choices could have been selected. 
Table 6. Distribution of respondents according to the duration of reported change in physical activity within different parameter groups

\begin{tabular}{|c|c|c|c|c|c|c|}
\hline \multicolumn{2}{|l|}{ Variable } & $\mathrm{n}$ & $\begin{array}{l}\text { Lasting } \\
\quad \%\end{array}$ & $\begin{array}{c}\text { Temporary } \\
\%\end{array}$ & $\begin{array}{c}\text { No change } \\
\%\end{array}$ & $p$ value* \\
\hline \multirow{3}{*}{ Age (years) } & $18-30$ & 91 & 29.7 & 27.5 & 42.9 & \multirow{3}{*}{0.189} \\
\hline & $31-50$ & 75 & 44.0 & 18.7 & 37.3 & \\
\hline & $51-65$ & 23 & 52.2 & 17.4 & 30.4 & \\
\hline \multirow{2}{*}{ Gender } & Men & 81 & 33.3 & 19.8 & 46.9 & \multirow{2}{*}{0.167} \\
\hline & Women & 108 & 41.7 & 25.0 & 33.3 & \\
\hline \multirow{4}{*}{ Total anthropometric risk score $(\mathrm{TAR})^{\star *}$} & 0 & 103 & 31.1 & 19.4 & 49.5 & \multirow{4}{*}{0.001} \\
\hline & 1 & 25 & 20.0 & 44.0 & 36.0 & \\
\hline & 2 & 32 & 56.3 & 15.6 & 28.1 & \\
\hline & 3 & 29 & 58.6 & 24.1 & 17.2 & \\
\hline \multirow{2}{*}{ Low physical activity level } & no & 111 & 42.3 & 12.6 & 45.0 & \multirow{2}{*}{$<0.001$} \\
\hline & yes & 78 & 32.1 & 37.2 & 30.8 & \\
\hline \multirow{2}{*}{ Low cardiorespiratory fitness *** } & no & 158 & 34.2 & 22.2 & 43.7 & \multirow{2}{*}{0.001} \\
\hline & yes & 25 & 68.0 & 24.0 & 8.0 & \\
\hline \multirow{4}{*}{ Lifestyle cumulative risk score } & 0 & 21 & 52.4 & 14.3 & 33.3 & \multirow{4}{*}{0.013} \\
\hline & 1 & 65 & 36.9 & 12.3 & 50.8 & \\
\hline & 2 & 73 & 41.1 & 26.0 & 32.9 & \\
\hline & $3-5$ & 30 & 23.3 & 43.3 & 33.3 & \\
\hline \multirow{6}{*}{$\begin{array}{l}\text { Self-perceived physical activity } \\
\text { (baseline) }\end{array}$} & 1 (very poor) & 16 & 43.8 & 37.5 & 18.8 & \multirow{6}{*}{0.040} \\
\hline & 2 (poor) & 21 & 38.1 & 28.6 & 33.3 & \\
\hline & 3 (low) & 40 & 30.0 & 40.0 & 30.0 & \\
\hline & 4 (moderate) & 51 & 43.1 & 17.6 & 39.2 & \\
\hline & 5 (more) & 45 & 37.8 & 11.1 & 51.1 & \\
\hline & 6 (high) and 7 (very high) & 16 & 37.5 & 6.3 & 56.3 & \\
\hline \multirow{4}{*}{ Subjectively perceived health (baseline) } & $\begin{array}{l}1 \text { (very poor) and } \\
2 \text { (poor) }\end{array}$ & 19 & 47.4 & 31.6 & 21.1 & \multirow{4}{*}{0.132} \\
\hline & 3 (satisfactory) & 54 & 42.6 & 29.6 & 27.8 & \\
\hline & 4 (good) & 87 & 33.3 & 19.5 & 47.1 & \\
\hline & 5 (very good) & 29 & 37.9 & 13.8 & 48.3 & \\
\hline
\end{tabular}

${ }^{*} \mathrm{p}$ value refers to $\mathrm{X}^{2}$ test over all answering categories; ${ }^{* *} \mathrm{TAR}: 0$ (BMI < 30 and normal $\left.\mathrm{BF} \%\right) ; 1$ (BMI < 25 and increased $\left.\mathrm{BF} \%\right) ; 2$ (BMI $25.0-29.99$ and increased $\mathrm{BF} \%$ ); $3(\mathrm{BMI} \geq 30) ;{ }^{* * *}$ percentages related to the number of subjects who attended cardiorespiratory fitness test

Table 7. Reported change in nutrition and change in self-perceived parameters SPA, SPH (baseline vs. feedback) within groups of respondents according to the duration of reported change in physical activity

\begin{tabular}{|c|c|c|c|c|c|}
\hline Variable & & $\begin{array}{c}\text { Lasting } \\
\begin{array}{c}(\mathrm{n}=72) \\
\%\end{array}\end{array}$ & $\begin{array}{c}\text { Temporary } \\
(n=43) \\
\%\end{array}$ & $\begin{array}{c}\text { No change } \\
(n=74) \\
\%\end{array}$ & $p$ value* \\
\hline \multirow{3}{*}{ Reported change in nutrition } & Lasting & 44.4 & 16.3 & 27.0 & \multirow{3}{*}{$<0.001$} \\
\hline & Temporary & 19.4 & 65.1 & 31.1 & \\
\hline & No change & 36.1 & 18.6 & 41.9 & \\
\hline \multirow{3}{*}{ Self-perceived physical activity (SPA) ${ }^{* *}$} & Reduction & 11.9 & 42.4 & 27.9 & \multirow{3}{*}{0.003} \\
\hline & Stable & 25.4 & 33.3 & 31.1 & \\
\hline & Increase & 62.7 & 24.2 & 41.0 & \\
\hline \multirow{3}{*}{ Subjectively perceived health $(\mathrm{SPH})^{\star *}$} & Reduction & 18.6 & 30.3 & 19.7 & \multirow{3}{*}{0.010} \\
\hline & Stable & 35.6 & 48.5 & 60.7 & \\
\hline & Increase & 45.8 & 21.2 & 19.7 & \\
\hline
\end{tabular}

${ }^{*} p$ value refers to $x^{2}$ test over all answering categories; ${ }^{* *}$ percentages related to the number of subjects with baseline-feedback comparison (lasting: $n=59$; temporary: $n=33$; no change: $n=61$ ) 
Table 8. Comparison of self-perceived parameters (SPA, SPH) within the group of respondents (baseline vs. feedback)

\begin{tabular}{|c|c|c|c|c|c|c|c|c|}
\hline \multirow{2}{*}{ Variable } & \multirow{2}{*}{$\begin{array}{c}\text { Number* } \\
n\end{array}$} & \multicolumn{2}{|c|}{ Baseline } & \multicolumn{2}{|c|}{ Feedback } & \multicolumn{2}{|c|}{ Difference $^{\star *}$} & \multirow{2}{*}{$p$ value $e^{\star \star \star}$} \\
\hline & & Mean & SD & Mean & SD & Mean & SD & \\
\hline SPA & 153 & 3.73 & 1.34 & 4.04 & 1.47 & 0.31 & 1.28 & 0.001 \\
\hline $\mathrm{SPH}$ & 153 & 3.69 & 0.85 & 3.77 & 0.89 & 0.08 & 0.94 & 0.150 \\
\hline
\end{tabular}

SPA - self-perceived physical activity; SPH - subjectively perceived health; Mean - average value of the parameter; SD - standard deviation of the parameter; *number of subjects with baseline-feedback comparison; ${ }^{* *}$ difference between values (baseline vs. feedback); ${ }^{* * *} p$ value refers to paired t-test

A comparison of self-perceived parameters in the whole group of respondents (baseline vs. feedback 6 months after examination) confirmed the increase in self-perceived physical activity $(\mathrm{p}=0.001)$ while subjectively perceived health did not change significantly (Table 8). When dividing respondents into groups according to the duration of reported PA changes, there was a significant increase in SPA and SPH among implementers of lasting changes in PA (Table 7).

\section{DISCUSSION}

Disposable intervention has low efficiency according to current knowledge in terms of lasting lifestyle changes (11). Its effect depends on the current stage in cycle of the individual change. These stages are relatively unstable over time, and significantly depend on actual life circumstances (27). People who underwent CPPE were at least partially motivated, seeking information or thinking about modifying their lifestyle. $20.4 \%$ of the sample has already implemented some lifestyle changes prior to the examination. This active attitude creates favourable conditions for the implementation of behavioural changes and significantly modifies characteristics of the sample. Despite the fact that CPPE was free, the level of socioeconomic status in terms of education was higher than in the general population (28). Two thirds of the subjects had a university degree, primary education was not represented. Probably this is due to higher motivation and responsibility for own health among more educated people and their better access to information sources (personal computers, the Internet). Information about CPPE was located on the website of the Masaryk University, and this fact probably favours a younger age category.

Given the data from population surveys in the Czech Republic in recent years $(7,14,15,29)$, there was a higher proportion of normal weight, but lower proportion of overweight and obesity in our sample. Overall, women were more likely to be of normal weight with an increased $\mathrm{BF} \%$. Conversely, athletic men with increased total weight due to above-average development of lean body mass but normal representation of body fat were often classified as overweight. This body composition cannot be considered as risky in relation to CVDs. Taking this into account the occurrence of overweight respondents with an increased $\mathrm{BF} \%$ (TAR 2 ) in terms of gender was almost balanced in our sample. On a population level, being overweight is more pronounced in men $(14,15,29)$, even though they are more devoted to intensive physical activity $(6,14)$. Similarly, this difference could be partially due to the proportion of men with the above-average development of lean body mass, especially in younger age categories. On the other hand, normal weight (but an increased proportion of body fat) may be misinterpreted as healthy especially among women.
A higher proportion of low PAL in women in our sample corresponds with findings that PA of all ages is generally higher in men (14). In the Czech population, more than $60 \%$ of adults across all age categories are assessed as "sedentary", the highest PA was observed in young adulthood but it is decreasing with age (6). On the contrary, in our sample the oldest age category had mostly sufficient PAL but was underrepresented. Subjects with low PAL (45.6\%) comprised the most numerous group, despite the high proportion of those with average or above average CRF. The exception was the category of young men with mostly sufficient or high PAL (not presented in Tables). The age structure of the sample (younger age) and the high proportion of athletes both men and women with above-average CRF is likely to be strongly influenced by inclusion of the CRF test. CPPE was obviously attractive for people with a positive attitude towards PA and was perceived as support for motivation in an effort to increase their PA.

In accordance with the results of a population survey EHIS, the consumption of F\&V in our sample was higher among women (14). Smoking was lower in our sample $(29,30)$. At least one of five assessed lifestyle risk factors was found in the majority of the examined. From this perspective, a reflection of their lifestyle has been useful. But, the sample appears to have lower risk in terms of behavioural characteristics, $\mathrm{CRF}$ and BMI in comparison with the general population. However, these characteristics - lower behavioural risk, higher SES, lower BMI, male gender, younger age - are typical for people with a tendency to overestimate their PA. From this perspective, participating in the examination allows for the correction of possible errors of self-esteem (9).

Compared to the general population, the prevalence of hypertension was low. This is likely due to age and lower risk profile, because these morbidities are frequent in individuals with overweight/obesity and older age groups $(7,29)$. In EHIS CR, there was an obvious dependence of subjectively perceived health on age; with increasing age, the proportion of people who perceive their health as good or very good decreased (14). Although our sample consisted of mostly younger people, their slightly lower $\mathrm{SPH}$, in comparison with the population (14), corresponds with the fact that they decided to actively search for and undergo preventive examinations.

Generally, there is an observed trend of decreasing willingness to participate in population surveys (31). Lower response rates in preventive programmes are reported in younger age groups (29). Generally, respondents have a higher socioeconomic status, better $\mathrm{SPH}$, and healthier lifestyle $(32,33)$. Also in our sample non-respondents of FQ had higher proportion of both low PAL and CRF than respondents. Therefore, the increase in PA for this group was necessary but also difficult. It can be assumed that nonrespondents were probably not successful in behavioural change 
and also not interested to report this failure. Another reason for non-responsiveness may be dissatisfaction with the examination itself, unwillingness to devote time to the feedback questionnaire or non-delivery of the questionnaire. Similarly, people with low PAL performed lasting changes less often. Conversely, people who were overweight or obese and have low CRF responded by increasing PA, which had more lasting character. This trend can be assessed as positive. However, it is necessary to take the possible social desirability bias to which these individuals are conditioned to into account (34). Correlation between reported improvement in PA and diet suggest a synergistic effect in people strongly motivated to change. This is in accordance with the expected higher efficiency of combined behavioural interventions (12).

An increase in PA after 6 months actually led to an improvement of subjective parameters (SPA, SPH), while temporary modification resulted more often in their worsening. This phenomenon may be related to a decrease in a self-assessment as a consequence of failure to increase PA. Surprisingly, those who declared no change, showed in $41.0 \%$ improvement in SPA, compared with the baseline. Due to the nature of the single intervention and the complexity and variability of influences that modify lifestyle, the impact of CPPE on the implementation of behavioural changes is necessarily limited. It is clear, that reporting changes and their duration are influenced by many other factors.

Our study has several limitations. The sample is not representative in terms of age distribution or the prevalence of risk factors. Participants were volunteers who were actively seeking testing; they were already partly motivated to change. Therefore, results cannot be generalized to the population. The main limitation is a reliance on self-report measures of lifestyle behaviours. Use of objective measurements to assess PA would provide more accurate estimates of changes in PA. Moreover, use of a standardized questionnaire would improve the comparability and validity, but it also would require greater time allocation, which may be perceived as a high participant burden (35). Since the inclusion of CRF test was relatively time consuming to maintain the duration of examination within 2 hours, we used a simple non-standardized questionnaire. The reliability of self-report measures can be affected by many subjective factors (memory, understanding the issue, subjective norms); social desirability bias (34); evaluation in a longer time gap through FQ, etc. FQ was sent out after 6 months, because our aim was to determine the duration of behavioural changes so that they could be regarded as effective in terms of health effect (11). The optimal time interval for sending FQ would be 1-2 months after CPPE and then resending in regular interval for the detection of changes. However, we feared a significant reduction of response rate when resending.

The effect of subjective factors is evident in the comparison of responses to two consecutive items of FQ. When answering the question "What was the specific contribution of the completed examinations?", $36.5 \%$ of respondents reported the decision to implement lifestyle changes directly motivated by examination; $24.9 \%$ reported support for previous decision to change. In the following direct question: "Have you been motivated by participating in the examination to change in any area of lifestyle?", $84.1 \%$ of respondents reported some change and more specifically $53.4 \%$ reported lasting change in any area of lifestyle (not indicated in Table). This difference may be due to social desirability bias, or a different perception of long-term benefits of examinations on the one hand and short-term minor changes in lifestyle on the other. However, the undeniable importance of subjective parameters should be emphasized. The parameter 'subjectively perceived health' has high predictive validity according to available literature. It is a stronger predictor of mortality than medical tests and examinations (36).

\section{CONCLUSIONS}

CPPE appears to be effective at the individual level in terms of providing information, supporting motivation and to some extent implementation of behavioural changes especially in physical activity behaviour. However, particularly people with lower health and behavioural risk profile underwent this examination. Testing of CRF is likely to modify characteristics of the sample in terms of age, gender, and risk profile, addressing men and younger age groups, which are otherwise less accessible for primary prevention. CRF can be used as part of preventive programmes targeting these specific groups. However, it should be noted that the inclusion of a CRF test in a primary preventive programme is less attractive and less effective for people with a higher behavioural risk profile or passive attitude towards health.

\section{Acknowledgement}

Supported by the project no. LQ1605 from the National Program of Sustainability II (MEYS CR).

\section{Conflict of Interests}

None declared

\section{REFERENCES}

1. Perk J, De Backer G, Gohlke H, Graham I, Reiner Z, Verschuren WM, et al.; Fifth Joint Task Force of the European Society of Cardiology and Other Societies on Cardiovascular Disease Prevention in Clinical Practice; European Association for Cardiovascular Prevention and Rehabilitation. European Guidelines on cardiovascular disease prevention in clinical practice (version 2012): The Fifth Joint Task Force of the European Society of Cardiology and Other Societies on Cardiovascular Disease Prevention in Clinical Practice (constituted by representatives of nine societies and by invited experts). Atherosclerosis. 2012 Jul;223(1):1-68.

2. Liu K, Daviglus ML, Loria CM, Colangelo LA, Spring B, Moller AC, et al. Healthy lifestyle through young adulthood and the presence of low cardiovascular disease risk profile in middle age: the Coronary Artery Risk Development in (Young) Adults (CARDIA) study. Circulation. 2012 Feb 28;125(8):996-1004.

3. Kline CE, Irish LA, Krafty RT, Sternfeld B, Kravitz HM, Buysse DJ, et al. Consistently high sports/exercise activity is associated with better sleep quality, continuity and depth in midlife women: the SWAN sleep study. Sleep. 2013 Sep 1;36(9):1279-88.

4. Dodd LJ, Al-Nakeeb Y, Nevill A, Forshaw MJ. Lifestyle risk factors of students: a cluster analytical approach. Prev Med. 2010 Jul;51(1):73-7.

5. Giles EL, Brennan M. Trading between healthy food, alcohol and physical activity behaviours. BMC Public Health. 2014 Nov 27;14:1231. doi: 10.1186/1471-2458-14-1231.

6. Hamřík Z, Sigmundová D, Kalman M, Pavelka J, Sigmund E. Physical activity and sedentary behaviour in Czech adults: results from the GPAQ study. Eur J Sport Sci. 2014;14(2):193-8.

7. Matoulek M. Obesity in the 21 st century in Czech Republic. Obes Facts. 2015;8 Suppl 1:18.

8. Vandelanotte C, Duncan MJ, Hanley C, Mummery WK. Identifying population subgroups at risk for underestimating weight health risks and overestimating physical activity health benefits. J Health Psychol. 2011 Jul;16(5):760-9. 
9. Watkinson C, van Sluijs EM, Sutton S, Hardeman W, Corder K, Griffin SJ. Overestimation of physical activity level is associated with lower BMI: a cross-sectional analysis. Int J Behav Nutr Phys Act. 2010 Sep 20;7:68. doi: 10.1186/1479-5868-7-68.

10. van Sluijs EM, Griffin SJ, van Poppel MN. A cross-sectional study of awareness of physical activity: associations with personal, behavioral and psychosocial factors. Int J Behav Nutr Phys Act. 2007 Nov 8;4:53.

11. Lin JS, O'Connor E, Whitlock EP, Beil TL. Behavioral counseling to promote physical activity and a healthful diet to prevent cardiovascular disease in adults: a systematic review for the U.S. Preventive Services Task Force. Ann Intern Med. 2010 Dec 7;153(11):736-50.

12. Johns DJ, Hartmann-Boyce J, Jebb SA, Aveyard P; Behavioural Weight Management Review Group. Diet or exercise interventions vs combined behavioral weight management programs: a systematic review and metaanalysis of direct comparisons. J Acad Nutr Diet. 2014 Oct;114(10):155768

13. Lee C, Owens RG. Men's health. In: Ayers S, Baum A, McManus C, Newman S, Wallston K, Weinman J, et al. editors. Cambridge handbook of psychology, health and medicine. 2nd ed. Cambridge: Cambridge University Press; 2007. p.132-6.

14. Institute of Health Information and Statistics of the Czech Republic (IHIS CR) [Internet]. European Health Interview Survey in the Czech Republic EHIS 2008. Prague: IHIS CR; 2011 [cited 2015 Apr 20]. Available from: http://www.uzis.cz/en/publications/european-health-interview-surveyczech-republic-ehis-2008. (In Czech)

15. Eurostat. European Health Interview Survey EHIS 2008 [Internet]. Eurostat [cited 2015 Dec 15]. Available from: http://ec.europa.eu/eurostat/ web/microdata/european-health-interview-survey.

16. Mitáš J, Ding D, Frömel K, Kerr J. Physical activity, sedentary behavior, and body mass index in the Czech Republic: a nationally representative survey. J Phys Act Health. 2014 Jul;11(5):903-7.

17. Fiala J, Brázdová Z. "Know and improve your health": symbiosis of preventive project and preventive medicine education at the medical faculty in Brno. Cent Eur J Public Health. 1996 Dec;4(4):257-62.

18. Fiala J, Brázdová Z. A comparison between the lifestyles of men and women - parents of school age children. Cent Eur J Public Health. 2000 May;8(2):94-100.

19. Rollnick S, Mason P, Butler C. Health behavior change: a guide for practitioners. New York: Elsevier Health Sciences; 1999.

20. Brázdová Z, Fiala J: Dietary guidelines in the Czech Republic. Acta Facultatis Medicae Universitatis Brunensis Masarykianae, 115. Brno: Masaryk University Brno; 1998.

21. Society for Nutrition. Dietary guidelines for the Czech Republic [Internet] Prague: Society for Nutrition; 2012 [cited 2015 Mar 23]. Available from: http://www.vyzivaspol.cz/vyzivova-doporuceni-pro-obyvatelstvo-ceskerepubliky/. (In Czech).

22. Haskell WL, Lee IM, Pate RR, Powell KE, Blair SN, Franklin BA, et al.; American College of Sports Medicine; American Heart Association. Physical activity and public health: updated recommendation for adults from the American College of Sports Medicine and the American Heart Association. Circulation. 2007 Aug 28;116(9):1081-93.
23. World Health Organization. Obesity and overweight [Internet]. Geneva: WHO [cited 2015 Feb 23]. Available from: http://www.who.int/mediacentre/factsheets/fs311/en/.

24. Bodystat Ltd. Bodystat 1500 [Internet]. [cited 2015 Dec 1]. Available from: http://www.bodystat.cz/1500.

25. Seliger V, Bartůněk Z. Mean values of various indices of physical fitness in the investigation of Czechoslovak population aged 12-55 years. International Biological Programme. Results of Investigations 1968-1974. Praha: C̆STV; 1976. (In Czech.)

26. IBM. IBM SPSS Statistics 22 Documentation [Internet]. [cited 2015 Dec 1]. Available from: http://www-01.ibm.com/support/docview. wss?uid=swg27038407.

27. Prochaska JO, DiClemente CC, Norcross JC. In search of how people change. Applications to addictive behaviors. Am Psychol. 1992 Sep;47(9):1102-14.

28. Czech Statistical Office (CZSO). Education of the entire population by age in 2013 [Internet]. Prague: CZSO [cited 2015 Mar 22]. Available from: https://www.czso.cz/csu/gender/4-gender_vzdelani.

29. Cífková R, Bruthans J, Adámková V, Jozífová V, Galovcová M, Wohlfart $\mathrm{P}$, et al. Prevalence of basic cardiovascular risk factors in the Czech population in the years 2006-2009: the Czech post-MONICA study. Cor Vasa. 2011; 53(4-5):220-9. (In Czech.)

30. Sochor O, Králíková E, Cífková R, Fiala J, Tomášková I, Kunzová Š, et al. Tobacco use and some characteristics of tobacco users. Preliminary results of "Kardiovize Brno 2030”. Cor Vasa. 2014;56(2):e118-26.

31. Cífková R, Skodová Z, Bruthans J, Adámková V, Jozífová M, Galovcová $\mathrm{M}$, et al. Longitudinal trends in major cardiovascular risk factors in the Czech population between 1985 and 2007/8. Czech MONICA and Czech post-MONICA. Atherosclerosis. 2010 Aug;211(2):676-81.

32. Van Loon AJ, Tijhuis M, Picavet HS, Surtees PG, Ormel J. Survey non-response in the Netherlands: effects on prevalence estimates and associations. Ann Epidemiol. 2003 Feb;13(2):105-10.

33. Hoebel J, Starker A, Jordan S, Richter M, Lampert T. Determinants of health check attendance in adults: findings from the cross-sectional German Health Update (GEDA) study. BMC Public Health. 2014 Sep 4;14:913. doi: 10.1186/1471-2458-14-913

34. Nederhof AJ. Methods of coping with social desirability bias: A review Eur J Soc Psychol. 1985 Jul/Sep;15(3):263-80.

35. Griffiths F, Lindenmeyer A, Powell J, Lowe P, Thorogood M. Why are health care interventions delivered over the internet? A systematic review of the published literature. J Med Internet Res. 2006 Jun 23;8(2):e10.

36. Benyamini Y, Idler EL. Community studies reporting association between self-rated health and mortality additional studies, 1995 to 1998 . Res Aging. 1999 Jan;21(3):392-401

Received April 29, 2015

Accepted in revised form December 18, 2015 